\title{
Straightforward synthesis of novel acene-based aryne precursors
}

\author{
Diego Rodríguez-Lojo, Diego Peña, Dolores Pérez*, Enrique Guitián
}

Centro de Investigación en Química Biolóxica e Materiais Moleculares (CIQUS) and Departamento de Química Orgánica, Facultade de Química, Universidade de Santiago de Compostela, 15782 Santiago de Compostela, Spain

Fax: +34881815704

E-mail: dolores.perez@usc.es

Received: The date will be inserted once the manuscript is accepted.

Dedicated to Prof. K. Peter C. Vollhardt

\begin{abstract}
Large polycyclic $o$-(trimethylsilyl)aryl triflates are easily obtained by reaction of commercially available bisaryne precursors with cyclopentadienones. The transformation involves controlled generation of one of the aryne functionalities, trapping by Diels-Alder reaction with a dienone, and chelotropic extrusion of CO. The newly synthetized triflates are precursors of acene-based arynes, including a didehydropentacene
\end{abstract}

Key words: arynes, polycyclic aromatic hydrocarbons (PAHs), acenes, Diels-Alder reaction, cycloadditions

In recent years the chemistry of arynes ${ }^{1}$ has experienced a significant resurgence mainly due to the availability of suitable methods for their generation. ${ }^{2}$ Most of the work published in the last decade used the fluoride-induced elimination from $o$ (trimethylsilyl)aryl triflates for the generation of arynes and hetarynes under mild conditions. This method, first described by Kobayashi et $a l^{3}$ and further developed by our group and others, spelled the discovery of novel reaction venues, including metalcatalyzed transformations, ${ }^{4,5}$ and facilitated the application of aryne chemistry to the synthesis of complex natural products ${ }^{6}$ and novel polycyclic aromatic systems. ${ }^{7}$

The preparation of simple $o$-(trimethylsilyl)aryl triflates is usually achieved in one or two synthetic steps from the corresponding $o$-bromophenols ${ }^{8}$ which, in many cases, are accessible by selective $o$ bromination of phenols. This procedure has been proved useful for the synthesis of a variety of benzyne, naphthyne or phenanthryne precursors, starting from easily available phenols, naphthols or phenanthrols. The preparation of slightly more complex polycyclic $o$-(trimethylsilyl)aryl triflates, such as the precursors of 2,3-triphenylyne ${ }^{9}, 2,3-$ biphenylyne $^{10}$ or corannulyne ${ }^{11}$ required longer, less efficient, synthetic routes. Here we report a straightforward method for the synthesis extended polycyclic $o$-(trimethylsilyl)aryl triflates by means of controlled [4+2] cycloadditions between cyclopentadienones and bistriflates $\mathbf{1}^{12}$ or $2^{13}$, which are formal precursors of the 1,4-benzodiyne and 2,6naphthodiyne respectively.

In the course of one of our research projects, focused to the synthesis of large PAHs with application in organic electronics, we recently reported the use of the masked bisbenzyne approach for the synthesis of the benzotriphenylyne precursor 3 . $^{14}$

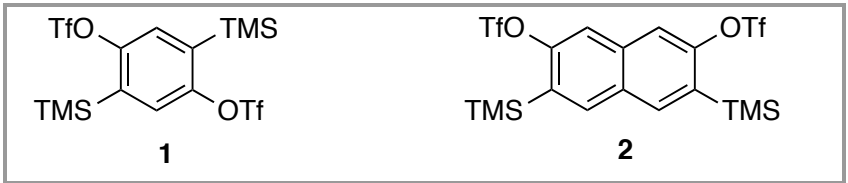

Figure 1

Thus, treatment of cyclopentadienone $4^{15}$ with 3 bromo-4-hydroxy-6-(trimethylsilyl)phenyl triflate (5) and TBAF afforded bromophenol 6, through a [4+2] cycloaddition of the dienone with aryne 7 , followed by chelotropic decarbonylation (Scheme 1, route $\mathbf{A}$ ).

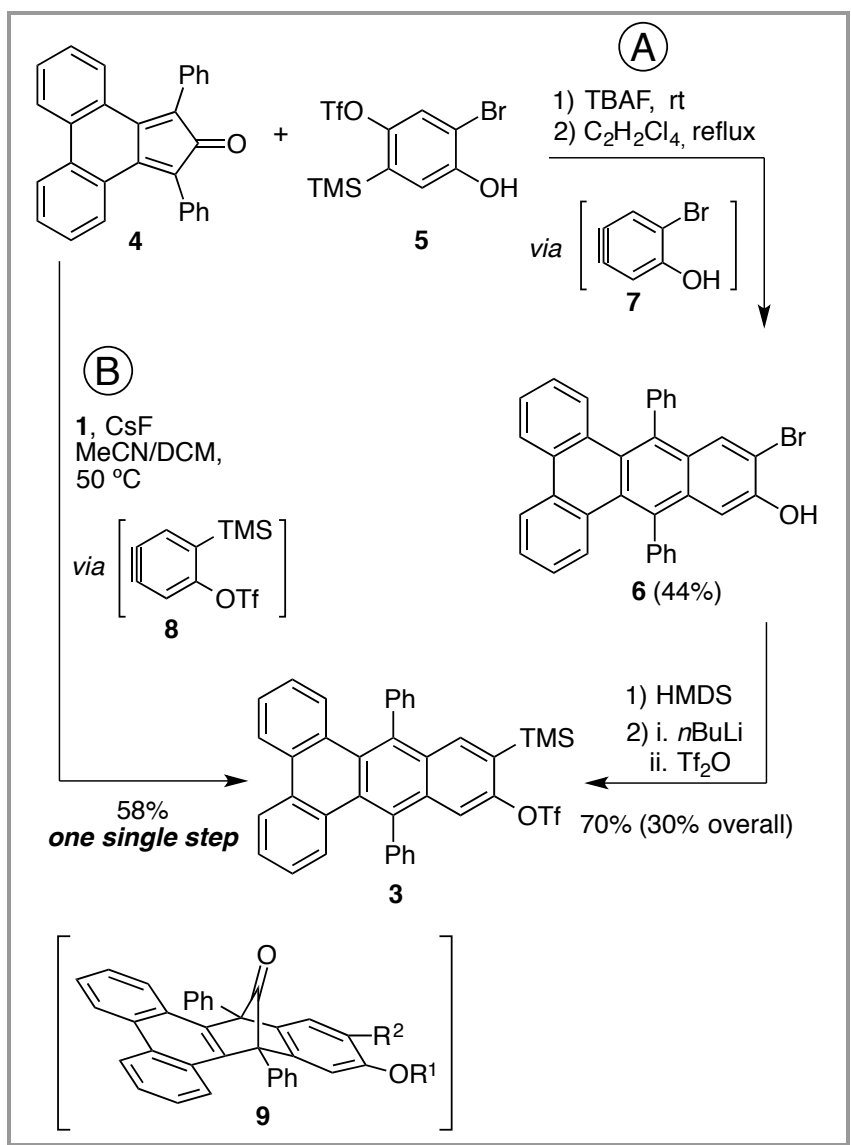

Scheme 1 Synthesis of 3: (A) two-step procedure (masked bisbenzyne approach $)^{14}$, (B) one step procedure using bistriflate 1

Transformation of $\mathbf{6}$ into the corresponding $o$ (trimethylsilyl)aryl triflate following our general established protocol, ${ }^{8}$ afforded benzotriphenylyne precursor $\mathbf{3}$ in $30 \%$ overall yield, which was 
subsequently subjected to Pd-catalyzed cyclotrimerization to build a clover-shaped 16-ring nanographene. In an attempt to synthetize $\mathbf{3}$ more efficiently, we explored the reaction of 4 with 2,5bis(trimethysilyl)-1,4-phenylene bis(triflate) (1), under controlled conditions for the selective generation and trapping of funtionalized monoaryne 8 . Successfully, we found that treatment of $\mathbf{1}$ with 120 mol\% anhydrous $\mathrm{CsF}$ in a $2: 1 \quad \mathrm{CH}_{3} \mathrm{CN} / \mathrm{CH}_{2} \mathrm{Cl}_{2}$ mixture, in the presence of 1,3-diphenyl-2Hcyclopenta[l]phenanthren-2-one (4), afforded triflate 3 in 58\% yield (Scheme 1, route B).

Table 1 Reaction of bistriflates $\mathbf{1}$ and $\mathbf{2}$ with cyclopentadienones

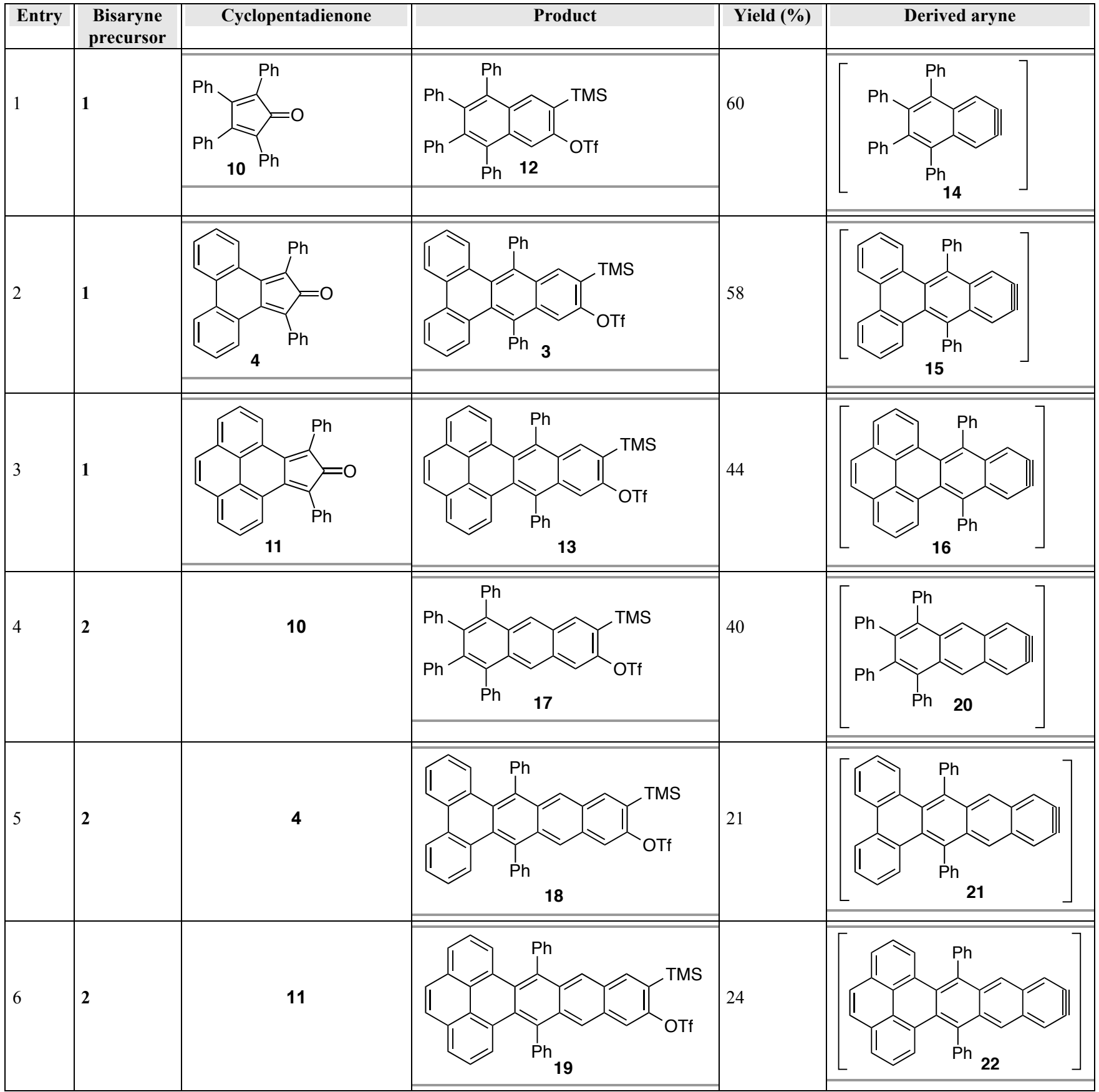

The choice of the solvent mixture and the use of CsF as fluoride source seem to be crucial for the success of this chemoselective transformation. ${ }^{17}$ On the one hand, $\mathrm{CsF}$ is only slightly soluble in these conditions, which allows the slow generation of the aryne. On the other hand, the lower solubility of the reaction products (with respect to the reagents) promotes their partial precipitation from the reaction mixture and minimizes the possibility of generating a second aryne.

The reaction proceeds via [4+2] cycloaddition reaction of monoaryne $\mathbf{8}$ to cyclopentadienone $\mathbf{4}$, followed by chelotropic extrusion of carbon monoxide. In some experiments, the intermediate cycloadduct 9 can be detected, and forced to evolve towards 3 upon additional heating. It is worth to note that bistriflate $\mathbf{1}$ had been previously used as 1,4- 
bisbenzyne synthon equivalent in a double DielsAlder reactions to build a stable twistacene ${ }^{12}$ but, to the best of our knowledge, no example of an efficient single fluoride-induced elimination to generate intermediate $\mathbf{8}$ had been described prior to our work. ${ }^{18}$

Application of the same protocol to the reaction of $\mathbf{1}$ with other dienones such as the commercially available 2,3,4,5-tetraphenylcyclopentadienone (10) and the easily accessible 9,11-diphenyl-10Hcyclopenta[e]pyren-10-one (11) ${ }^{19}$ afforded the corresponding $o$-(trimethylsilyl)aryl triflates 12 and 13 in $60 \%$ and $44 \%$ yields, respectively (Table 1, entries 1 and 3 ). As mentioned above, compound $\mathbf{3}$ is a convenient precursor of the dibenzofused 2,3didehydroanthracene $\mathbf{1 5}$ as demonstrated in the synthesis of a [16]cloverphene. ${ }^{14}$ On the other hand, the tetraphenylnapthyne 14, which can be generated from 12, and the bisbenzotetracyne 16, available through 13, had been obtained before from other precursors $^{20,21}$ and employed in Diels-Alder reactions for the synthesis of stable, phenyl substituted and/or benzoannulated large acenes.

In order to extend the polycyclic aryne core towards larger acenes, we explored the reactions of the 2,6naphthodiyne precursor 2. Satisfyingly, treatment of $\mathbf{2}$ with $120 \mathrm{~mol} \% \mathrm{CsF}$ in the presence of dienes 4, 10 and 11 under similar controlled conditions, afforded the corresponding $o$-(trimethylsilyl)aryl triflates 17-19 as major products, in reasonable yields (Table 1, entries 4-6). ${ }^{22}$ Remarkably, triflate $\mathbf{1 9}$ is a stable, functionalized pentacene ${ }^{23}$ and a potential precursor of the large pentacene-based aryne 22. The UV/Vis spectra of 19 showed the lowest-energy absorption band at $\lambda_{\text {onset }}=484 \mathrm{~nm}$, with a band gap of $2.56 \mathrm{eV}$, slightly lower than the HOMO-LUMO gap of a previously described pyrene-terminated pentacene. ${ }^{24}$ On the other hand, cyclic voltammetry $(\mathrm{CV})$ showed a reversible reduction process at $\mathrm{E}_{1 / 2}{ }^{\text {red }}=-1.48 \mathrm{~V}$.

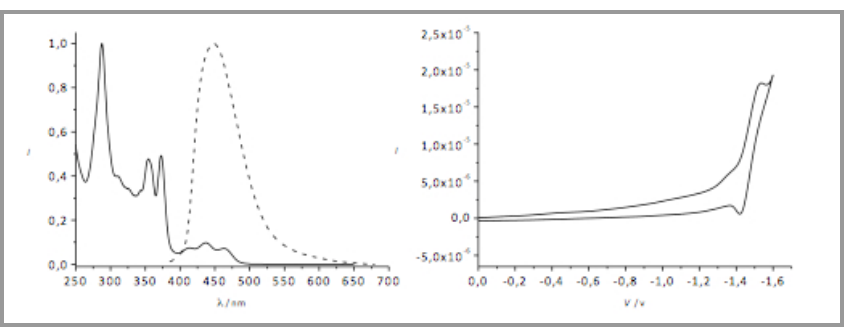

Figure 2 Electronic properties of pentacene 19: (a) absorption (solid line) and emission (dashed line) spectra in $\mathrm{CH}_{2} \mathrm{Cl}_{2}$. (b) Cyclic voltammogram in $\mathrm{CH}_{2} \mathrm{Cl}_{2} / 0.1 \mathrm{M} \mathrm{Bu}_{4} \mathrm{NPF}_{6}$ using $\mathrm{AgCl} / \mathrm{Ag}$ as the reference electrode

In conclusion, an expeditious method for the synthesis of novel polycyclic $o$-(trimethylsilyl)aryl triflates, based on the controlled reaction of a formal bisaryne precursor with cyclopentadienones has been described. The application of these compounds to the synthesis of novel acene, phene and starphene dervivatives by means of aryne cycloaddition reactions will be reported in due course.

Supporting Information for this article is available online at http://www.thiemeconnect.com/products/ejournals/journal/10.1055/s00000083 .

\section{Acknowledgment}

Financial support from the Spanish Ministry of Science and Competitiveness (MINECO, CTQ2013-44142-P and MAT2013-46593-C6-6-P), the European Union (Project PAMS, contract no. 610446), Xunta de Galicia (GPC2014/25) and FEDER is gratefully acknowledged. D.R.-L. thanks the Spanish Ministry of Education for the award of an FPU fellowship.

\section{References}

(1) General reviews on aryne chemistry: (a) Hoffmann, R. W. Dehydrobenzene and Cycloalkynes, Academic Press, New York, 1967. (b) Hart, H. Arynes and Heteroarynes Suppl. C2: The Chemistry of Triple-Bonded Functional Groups (Eds.: S. Patai, Z. Rappoport), Wiley, Chichester, 1994, p. 1017. (c) Pellisier, H.; Santelli, M.; Tetrahedron 2003, 59, 701; e) R. Sanz, Org. Prep. Proc. Int. 2008, 40, 215.

(2) Kitamura, T. Aust. J. Chem. 2010, 98, 987

(3) Himeshima, Y.; Sonoda, T.; Kobayashi, H. Chem. Lett. 1983,1211.

(4) Representative metal-catalyzed transformations involving arynes: (a) Peña, D.; Escudero, S.; Pérez, D.; Guitián, E.; Castedo, L. Angew. Chem. Int. Ed. 1998 , 37, 2659; (b) Peña, D.; Pérez, D.; Guitián, E.; Castedo, L. J. Am. Chem. Soc. 1999, 121, 5827; (c) Yokinawa, E.; Yamamoto, Y. Angew. Chem. Int. Ed. 2000, 39,173. (d) Peña, D.; Pérez, D.; Guitián, E. Palladium-catalyzed cycloaddition reactions of arynes in Palladium in Organic Synthesis vol. 14 (Ed.: J. Tsuji), Springer Verlag, Weinheim, 2005, p. 109; (e) Liu, Z.; Larock R. C. J. Org. Chem. 2007, 72, 223; (f) Yoshida, H.; Ohshita, J.; Kunai, A. Bull. Chem. Soc. Jpn. 2010, 83, 199; (g) Worlikar, S. A.; Larock, R. C. Current. Org. React. 2011, 15, 3214.

(5) Representative novel metal-free transformations: (a) Peña, D.; Pérez, D.; Guitián, E Angew. Chem. Int. Ed. 2006, 45, 3579 (b) Bhunia, A.; Yetra, S. R.; Biju, A. T. Chem. Soc. Rev., 2012, 41, 3140 (c) Bhunia, A; Biju, A. T. Synlett 2014, 608. (d) Criado, A.; Vilas-Varela, M.; Cobas, A.; Pérez, D.; Peña, D.; Guitián, E. J. Org. Chem. 2013, 78, 12637; (e) Dubrovskiy, A. V.; Markina, N.A.; Larock, R. C. Org. Biomol. Chem. 2013, $11,191$.

(6) (a) Tadross, P. M.; Stoltz, B. M.; Chem. Rev. 2012, 112, 3550; (b) Gampe, C. M.; Carreira, E. M. Angew. Chem. Int. Ed. 2012, 51, 3766.

(7) (a) Pérez, D.; Peña, D.; Guitián, E. Eur. J. Org. Chem. 2013, 5981; (b) Li, J.; Zhang, Q. Synlett 2013, 24, 686.

(8) Peña, D.; Cobas, A.; Pérez, D.; Guitián, E.; Castedo, L. Synthesis 2002, 1454.

(9) Romero, C.; Peña, D.; Pérez, D.; Guitián, E. Chem. Eur. J. 2006, 12, 5677.

(10) Iglesias, B.; Cobas, A.; Pérez, D.; Guitián, E.; Vollhardt, K. P. C. Org. Lett. 2004, 6, 3557.

(11) Sygula, A.; Sygula, R.; Kobryn, L. Org. Lett. 2008, 10, 3927. 
(12) Duong, H. M.; Bendikov, M.; Steiger, D.; Zhang, Q.; Sonmez, G.; Yamada, J.; Wudl, F. Org. Lett. 2003, 5, 4433.

(13) Kitamura, C.; Abe, Y.; Ohara, T.; Yoneda, A.; Kawase, T.; Kobayashi, T.; Naito, H.; Komatsu, T. Chem. Eur. J. 2010, 16, 890 .

(14) Alonso, J. M.; Díaz-Álvarez, A. E.; Criado, A.; Peña, D.; Pérez, D.; Guitián, E. Angew. Chem. Int. Ed. 2012, $51,173$.

(15) Wooi, G. Y.; White, J. M. Org. Biomol. Chem. 2005, 3, 972.

(16) General procedure: To a solution of bisaryne precursor (1 or 2$)$ and cyclopentadienone $(4,10$ or $11,110 \mathrm{~mol} \%)$, in $2: 1 \mathrm{CH}_{3} \mathrm{CN} / \mathrm{CH}_{2} \mathrm{Cl}_{2}$, finely powdered anhydrous $\mathrm{CsF}$ $(120 \mathrm{~mol} \%)$ was added, and the mixture was stirred at $50{ }^{\circ} \mathrm{C}$ under argon atmosphere for $12 \mathrm{~h}$. The solvent was evaporated under reduced pressure and the resulting mixture was purified by column chromatography $\left(\mathrm{SiO}_{2}\right.$, hexanes $/ \mathrm{CH}_{2} \mathrm{Cl}_{2}$ ) to isolate the corresponding $O$ (trimethylsilyl)aryl triflate. In some experiments, the intermediate Diels-Alder adduct (e.g. 9) was detected in other fractions eluted from the column, which were collected, concentrated under vacuum, dissolved in tetrachloroethane and refluxed for $12 \mathrm{~h}$. Evaporation of the solvent under vacuum and column chromatography $\left(\mathrm{SiO}_{2}\right.$, hexanes $\left./ \mathrm{CH}_{2} \mathrm{Cl}_{2}\right)$ of the residue afforded an additional portion of $o$-(trimethylsilyl)aryl triflate.

9,14-Diphenyl-12-(trimethylsilyl)benzo[ $f$ ]tetraphen11-yl trifluoromethanesulfonate (3): Reaction of 1,3diphenyl-2H-cyclopenta[l]phenanthren-2-one $\quad \mathbf{4}, 243$ $\mathrm{mg}, 0.637 \mathrm{mmol})$, 2,5-bis(trimethylsilyl)-1,4-phenylene bis(trifluoromethanesulfonate) $(1,300 \mathrm{mg}, 0.579 \mathrm{mmol})$ and $\mathrm{CsF}(105 \mathrm{mg}, 0.695 \mathrm{mmol})$ in $2: 1 \mathrm{CH}_{3} \mathrm{CN} / \mathrm{CH}_{2} \mathrm{Cl}_{2}$ $(9 \mathrm{~mL})$ afforded $3(218 \mathrm{mg}, 58 \%)$ as a greenish solid; m.p. $219-221^{\circ} \mathrm{C} .{ }^{1} \mathrm{H}$ NMR (250 MHZ, $\left.\mathrm{CDCl}_{3}\right): \delta=8.29$ $(\mathrm{d}, J=7.3 \mathrm{~Hz}, 2 \mathrm{H}), 8.13(\mathrm{~s}, 1 \mathrm{H}), 7.85(\mathrm{~s}, 1 \mathrm{H}), 7.59$ (d, $J$ $=8.5 \mathrm{~Hz}, 2 \mathrm{H}), 7.54(\mathrm{~m}, 5 \mathrm{H}), 7.54(\mathrm{~m}, 5 \mathrm{H}), 7.41-7.36$ $(\mathrm{m}, 2 \mathrm{H}), 7.04-6.98(\mathrm{~m}, 2 \mathrm{H}), 0.33(\mathrm{~s}, 9 \mathrm{H}) \mathrm{ppm} .{ }^{13} \mathrm{C}$ NMR (63 MHz, $\left.\mathrm{CDCl}_{3}\right): \delta=152.6(\mathrm{C}), 140.9$ (C), 140.5 (C), $136.8(\mathrm{CH}), 135.4(\mathrm{C}), 135.2$ (C), 132.7 (C), 132.3 (2CH), 132.2 (C), $132.1(2 \mathrm{CH}), 132.1$ (C), 131.9 (C), 130.8 (C), $130.6(\mathrm{C}), 130.55$ (C), $130.50(\mathrm{CH}), 130.4$ $(\mathrm{CH}), 129.8(\mathrm{C}), 129.3(\mathrm{C}), 129.1(2 \mathrm{CH}), 129.0(2 \mathrm{CH})$, $128.0(\mathrm{CH}), 127.9(\mathrm{CH}), 127.3(\mathrm{CH}), 127.2(\mathrm{CH}), 125.9$ $(2 \mathrm{CH}), 123.3(2 \mathrm{CH}), 115.7(\mathrm{CH}),-0.8\left(3 \mathrm{CH}_{3}\right) \mathrm{ppm}$. MS (EI), $m / z$ (\%): 650 (30). HRMS (EI): $\mathrm{m} / \mathrm{z}$ calcd for $\mathrm{C}_{38} \mathrm{H}_{29} \mathrm{~F}_{3} \mathrm{O}_{3} \mathrm{SSi}$ : 650.1559 ; found: 650.1563 .

5,6,7,8-Tetraphenyl-3-(trimethylsilyl)naphthalen-2-yl trifluoromethanesulfonate (12): Reaction of 2,3,4,5tetraphenylcyclopenta-2,4-dien-1-one $\quad(\mathbf{1 0}, 329 \mathrm{mg}$, $0.858 \mathrm{mmol}), \quad 2,5$-bis(trimethylsilyl)-1,4-phenylene bis(trifluoromethanesulfonate) $(1,404 \mathrm{mg}, 0.780 \mathrm{mmol})$ and $\mathrm{CsF}$ (142 mg, $0.936 \mathrm{mmol}$ ) in $2: 1 \mathrm{CH}_{3} \mathrm{CN} / \mathrm{CH}_{2} \mathrm{Cl}_{2}$ $(11 \mathrm{~mL})$ afforded $12(305 \mathrm{mg}, 60 \%)$ as a white solid; m.p. $249-251{ }^{\circ} \mathrm{C} .{ }^{1} \mathrm{H}$ NMR (300 MHZ, $\left.\mathrm{CDCl}_{3}\right): \delta=7.89$ $(\mathrm{s}, 1 \mathrm{H}), 7.59(\mathrm{~s}, 1 \mathrm{H}), 7.32-7.20(\mathrm{~m}, 10 \mathrm{H}), 6.92-6.87(\mathrm{~m}$, $10 \mathrm{H}), 0.30(\mathrm{~s}, 9 \mathrm{H}) \mathrm{ppm} .{ }^{13} \mathrm{C} \mathrm{NMR}\left(75 \mathrm{MHz}, \mathrm{CDCl}_{3}\right): \delta$ $=152.8(\mathrm{C}), 140.7(\mathrm{C}), 139.9(4 \mathrm{C}), 138.7(2 \mathrm{C}), 138.5$ (C), $138.3(\mathrm{C}), 136.9(\mathrm{CH}), 133.4(\mathrm{C}), 131.1(3 \mathrm{CH})$, $131.1(4 \mathrm{CH}), 130.9(2 \mathrm{CH}), 130.5(\mathrm{C}), 127.7(2 \mathrm{CH})$, $127.6(2 \mathrm{CH}), 126.9(\mathrm{CH}), 126.7(4 \mathrm{CH}), 125.7(\mathrm{CH})$, $125.6(\mathrm{CH}), 118.4\left(\mathrm{q}, J=320 \mathrm{~Hz}, \mathrm{CF}_{3}\right), 116.3(\mathrm{CH})$, $0.9\left(3 \mathrm{CH}_{3}\right) \mathrm{ppm}$. MS (EI), $\mathrm{m} / \mathrm{z}(\%): 652.3\left(\mathrm{M}^{+}, 70\right)$, 504.3 (14), 293.3 (41), 147.1 (43). HRMS (EI): $\mathrm{m} / \mathrm{z}$ calcd for $\mathrm{C}_{38} \mathrm{H}_{31} \mathrm{~F}_{3} \mathrm{O}_{3} \mathrm{SSi}$ : 652.1715 ; found: 652.1713 .

9,14-Diphenyl-12-

(trimethylsilyl)dibenzo[de,qr]tetracen-11-yl

trifluoromethanesulfonate (13): Reaction of 9,11diphenyl-10H-cyclopenta[e]pyren-10-one $(\mathbf{1 1}, 100 \mathrm{mg}$,
$0.246 \mathrm{mmol}), \quad$ 2,5-bis(trimethylsilyl)-1,4-phenylene bis(trifluoromethanesulfonate) (1, $116 \mathrm{mg}, 0.224 \mathrm{mmol})$ and $\mathrm{CsF}(41 \mathrm{mg}, 0.269 \mathrm{mmol})$ in $2: 1 \mathrm{CH}_{3} \mathrm{CN} / \mathrm{CH}_{2} \mathrm{Cl}_{2}(9$ $\mathrm{mL}$ ) afforded $13(66 \mathrm{mg}, 40 \%)$ as a yellow solid; m.p. 279-281 ${ }^{\circ} \mathrm{C}$. ${ }^{1} \mathrm{H}$ NMR $\left(500 \mathrm{MHZ}, \mathrm{CDCl}_{3}\right): \delta=8.11$ (s, $1 \mathrm{H}), 7.90-7.85(\mathrm{~m}, 6 \mathrm{H}), 7.82(\mathrm{~s}, 1 \mathrm{H}), 7.56-7.48(\mathrm{~m}$, $10 \mathrm{H}), 7.35$ (td, $J=7.9,2.2 \mathrm{~Hz}, 2 \mathrm{H}), 0.32(\mathrm{~s}, 9 \mathrm{H}) \mathrm{ppm}$. ${ }^{13} \mathrm{C}$ NMR (126 MHz, $\left.\mathrm{CDCl}_{3}\right): \delta=153.0(\mathrm{C}), 141.7(\mathrm{C})$, $141.4(\mathrm{C}), 137.3(\mathrm{CH}), 136.9(\mathrm{C}), 136.6(\mathrm{C}), 133.2(\mathrm{C})$, $132.4(2 \mathrm{CH}), 132.2(2 \mathrm{CH}), 131.0(\mathrm{C}), 131.0(\mathrm{C}), 130.99$ (C) 130.97 (C), 130.3 (C), 130.2 (C), 130.1 (C), 129.9 (C), $129.5(2 \mathrm{CH}), 129.4(2 \mathrm{CH}), 129.1(\mathrm{CH}), 129.0$ $(\mathrm{CH}), 128.2(\mathrm{CH}), 128.1(\mathrm{CH}), 127.1(\mathrm{CH}), 127.1(\mathrm{CH})$, $126.6(\mathrm{CH}), 126.4(\mathrm{CH}), 126.2(\mathrm{C}), 126.1(\mathrm{C}), 125.1$ $(\mathrm{CH}), 125.0(\mathrm{CH}), 118.5\left(\mathrm{q}, J=325 \mathrm{~Hz}, \mathrm{CF}_{3}\right), 116.1$ $(\mathrm{CH}),-0.6\left(3 \mathrm{CH}_{3}\right) \mathrm{ppm}$. MS (EI), $\mathrm{m} / z(\%): 674(100$, $\mathrm{M}+), 541$ (53), 448 (16).

5,6,7,8-Tetraphenyl-3-(trimethylsilyl)anthracen-2-yl trifluoromethanesulfonate (17): Reaction of 2,3,4,5tetraphenylcyclopenta-2,4-dien-1-one (10, $126 \mathrm{mg}$, $0.329 \mathrm{mmol}), \quad 3,6$-bis(trimethylsilyl)-naphthalene-2,7diyl bis(trifluoromethanesulfonate) $(\mathbf{2}, 115 \mathrm{mg}, 0.299$ $\mathrm{mmol}$ ) and $\mathrm{CsF}$ (55 $\mathrm{mg}, \quad 0.359 \mathrm{mmol}$ ) in 2:1 $\mathrm{CH}_{3} \mathrm{CN} / \mathrm{CH}_{2} \mathrm{Cl}_{2}(9 \mathrm{~mL})$ afforded $17(84 \mathrm{mg}, 40 \%)$ as a white solid; m.p. 223-225 ${ }^{\circ} \mathrm{C} .{ }^{1} \mathrm{H}$ NMR (300 MHZ, $\left.\mathrm{CDCl}_{3}\right): \delta=8.27(\mathrm{~s}, 1 \mathrm{H}), 8.22(\mathrm{~s}, 1 \mathrm{H}), 8.04(\mathrm{~s}, 1 \mathrm{H}), 7.82$ $(\mathrm{s}, 1 \mathrm{H}), 7.39-7.21(\mathrm{~m}, 10 \mathrm{H}), 7.01-6.78(\mathrm{~m}, 10 \mathrm{H}), 0.43$ (s, 9H) ppm. ${ }^{13} \mathrm{C}$ NMR $\left(75 \mathrm{MHz}, \mathrm{CDCl}_{3}\right): \delta=152.4(\mathrm{C})$, 140.5 (2C), 139.91 (C), 139.59 (C), 139.52 (C), 139.4 (C), $139.0(\mathrm{CH}), 138.6(\mathrm{C}), 138.3(\mathrm{C}), 132.1(\mathrm{C}), 131.7$ (C), $131.6(\mathrm{CH}), 131.6(\mathrm{CH}), 131.4(\mathrm{CH}), 129.6(\mathrm{C})$, $128.1(\mathrm{CH}), 128.0(\mathrm{CH}), 127.0(\mathrm{CH}), 126.9(\mathrm{CH}), 126.9$ $(\mathrm{CH}), 126.8(\mathrm{CH}), 126.4(\mathrm{CH}), 125.7(\mathrm{CH}), 116.2(\mathrm{CH})$, $-0.4\left(3 \mathrm{CH}_{3}\right) \mathrm{ppm}$. MS (MALDI-TOF), $m / z 702\left(\mathrm{M}^{+}\right)$.

\section{9,16-Diphenyl-13-}

(trimethylsilyl)dibenzo[a,c]tetracen-12-yl

trifluoromethanesulfonate (18): Reaction of 1,3diphenyl-2H-cyclopenta[l]phenanthren-2-one $\quad \mathbf{( 4 ,}, 148$ $\mathrm{mg}, 0.388 \mathrm{mmol}), 3,6$-bis(trimethylsilyl)-naphthalene2,7-diyl bis(trifluoromethanesulfonate) $(2,200 \mathrm{mg}$, $0.353 \mathrm{mmol}$ ) and $\mathrm{CsF}(65 \mathrm{mg}, 0.424 \mathrm{mmol})$ in $2: 1$ $\mathrm{CH}_{3} \mathrm{CN} / \mathrm{CH}_{2} \mathrm{Cl}_{2}(9 \mathrm{~mL})$ afforded $\mathbf{1 8}(50 \mathrm{mg}, 21 \%)$ as a yellow solid. ${ }^{1} \mathrm{H}$ NMR (500 MHZ, $\left.\mathrm{CDCl}_{3}\right): \delta=8.51$ (s, $1,8.46(\mathrm{~s}, 1,8.18(\mathrm{~d}, J=7.9 \mathrm{~Hz}, 2 \mathrm{H}), 8.05(\mathrm{~s}, 1 \mathrm{H})$, $7.84(\mathrm{~s}, 1 \mathrm{H}), 7.66-7.54(\mathrm{~m}, 10 \mathrm{H}), 7.42(\mathrm{t}, J=8.0 \mathrm{~Hz}$, $2 \mathrm{H}), 7.32(\mathrm{t}, J=7.5 \mathrm{~Hz}, 2 \mathrm{H}), 6.95(\mathrm{t}, J=7.7 \mathrm{~Hz}, 2 \mathrm{H})$, $0.43(\mathrm{~s}, 9 \mathrm{H}) \mathrm{ppm} .{ }^{13} \mathrm{C}$ NMR $\left(126 \mathrm{MHz}, \mathrm{CDCl}_{3}\right): \delta=$ 152.4 (C), $141.6(2 \mathrm{C}), 141.5(2 \mathrm{C}), 139.0(\mathrm{CH}), 135.4$ (C), $135.0(\mathrm{C}), 133.0(2 \mathrm{CH}), 133.0(2 \mathrm{CH}), 132.6(\mathrm{C})$, 132.6 (C), 131.8 (C), 131.5 (C), 131.5 (C), 131.4 (C), $131.2(\mathrm{C}), 131.0(\mathrm{CH}), 130.9(\mathrm{CH}), 130.0(\mathrm{C}), 129.6$ $(2 \mathrm{CH}), 129.5(2 \mathrm{CH}), 128.3(\mathrm{CH}), 128.2(\mathrm{CH}), 128.06$ (C), $127.6(\mathrm{CH}), 127.6(\mathrm{CH}), 126.5(\mathrm{CH}), 126.3(\mathrm{CH})$, $126.0(\mathrm{CH}), 123.7(\mathrm{CH}), 118.2\left(\mathrm{q}, J=321 \mathrm{~Hz}, \mathrm{CF}_{3}\right)$, $116.3(\mathrm{CH}),-0.4(\mathrm{CH} 3) \mathrm{ppm}$. MS (MALDI-TOF), $\mathrm{m} / \mathrm{z}$ $700\left(\mathrm{M}^{+}\right)$.

9,16-Diphenyl-13-

(trimethylsilyl)dibenzo[de, $u v]$ pentacen-12-yl

trifluoromethanesulfonate (19): Reaction of 9,11diphenyl-10H-cyclopenta[e]pyren-10-one $(\mathbf{1 1}, 118 \mathrm{mg}$, $0.290 \mathrm{mmol}), \quad 3,6$-bis(trimethylsilyl)-naphthalene-2,7diyl bis(trifluoromethanesulfonate) $(2,150 \mathrm{mg}, 0.264$ $\mathrm{mmol}$ ) and $\mathrm{CsF}$ (48 $\mathrm{mg}, \quad 0.317 \mathrm{mmol})$ in $2: 1$ $\mathrm{CH}_{3} \mathrm{CN} / \mathrm{CH}_{2} \mathrm{Cl}_{2}(9 \mathrm{~mL})$ afforded $19(46 \mathrm{mg}, 24 \%)$ as a yellow solid. ${ }^{1} \mathrm{H}$ NMR (500 MHZ, $\left.\mathrm{CDCl}_{3}\right): \delta=8.52(\mathrm{~s}$, $1 \mathrm{H}), 8.47(\mathrm{~s}, 1 \mathrm{H}), 8.06(\mathrm{~s}, 1 \mathrm{H}), 7.85(\mathrm{~m}, 3 \mathrm{H}), 7.82(\mathrm{~d}, J$ $=7.7 \mathrm{~Hz}, 2 \mathrm{H}), 7.73(\mathrm{t}, J=8.4 \mathrm{~Hz}, 2 \mathrm{H}), 7.65-7.57(\mathrm{~m}$, $10 \mathrm{H}), 7.29$ (td, $J=7.9,1.2 \mathrm{~Hz}, 2 \mathrm{H}), 0.44(\mathrm{~s}, 9 \mathrm{H}) \mathrm{ppm}$. ${ }^{13} \mathrm{C}$ NMR $\left(126 \mathrm{MHz}, \mathrm{CDCl}_{3}\right): \delta=152.4(\mathrm{C}), 142.1(\mathrm{C})$, 
142.0 (C), $139.1(\mathrm{CH}), 136.6(\mathrm{C}), 136.6(\mathrm{C}), 132.8$ $(2 \mathrm{CH}), 132.76(2 \mathrm{CH}), 132.0(\mathrm{C}), 131.6(\mathrm{C}), 131.6(\mathrm{C})$, 131.3 (C), 131.0 (C), 130.9 (C), 130.4 (C), 130.4 (C), $130.1(\mathrm{C}), 129.7(\mathrm{CH}), 129.6(\mathrm{CH}), 129.4(\mathrm{CH}), 129.3$ $(\mathrm{CH}), 128.2(\mathrm{CH}), 128.1(\mathrm{CH}), 127.0(\mathrm{CH}), 127.0(\mathrm{CH})$, $126.7(\mathrm{CH}), 126.6(\mathrm{CH}), 126.6(\mathrm{CH}), 126.4(\mathrm{C}), 126.3$ $(\mathrm{C}), 126.1(\mathrm{CH}), 125.1(\mathrm{CH}), 125.1(\mathrm{C}), 118.2(\mathrm{q}, J=$ $\left.322 \mathrm{~Hz}, \mathrm{CF}_{3}\right), 116.29(\mathrm{CH}), 116.28(\mathrm{CH}),-0.43(3 \mathrm{CH} 3)$ ppm. MS (MALDI-TOF), $m / z 724\left(\mathrm{M}^{+}\right)$.

(17) The use of neat $\mathrm{CH}_{3} \mathrm{CN}$ or $\mathrm{CH}_{2} \mathrm{Cl}_{2}$, resulted in lower conversions due to the poor solubility of either the diene (in $\mathrm{CH}_{3} \mathrm{CN}$ ) or the $\mathrm{CsF}$ (in $\mathrm{CH}_{2} \mathrm{Cl}_{2}$ ). On the other hand the use of TBAF as the fluoride source afforded lower yields of the expected triflates.

(18) In parallel to the work described in this article, we also studied the generation of a naphtho[1,2,3,4-ghi]perylyne precursor by reaction of $\mathbf{8}$ with perylene: Schuler, B.; Collazos, S.; Gross, L.; Meyer, G.; Pérez, D.; Guitián, E.; Peña, D.; Angew. Chem. Int. Ed. 2014, 53, 9004.

(19) Pascal Jr., R. A.; Mcmillan, W. D.; Engen, D. Van; Eason, R. G. J. Am. Chem. Soc. 1987, 109, 4660.

(20) Miao, Q.; Chi, X.; Xiao, S.; Zeis, R.; Lefernfeld, M.; Siegrist, T.; Steigerwald, M. L.; Nuckolls, C. J. Am. Chem. Soc. 2006, 128, 1340.

(21) (a) Zhang, Q.; Divayana, Y.; Xiao, J.; Wang, Z.; Tiekink, E. R. T; Doung, H. M.; Zhang, H.; Boey, F.; Sun, X. W.; Wudl, F. Chem. Eur. J. 2010, 16, 7422. (b) J. Xiao, S. Liu, Y. Liu, L. Ji, X. Liu, H. Zhang, X. Sun, Q. Zhang, Chem. Asian J. 2012, 7, 561. (c) J. Xiao, H. M. Duong, Y. Liu, W. Shi, L. Ji, G. Li, S. Li, X.- W. Liu, J. Ma, F. Wudl, Q. Zhang, Angew. Chem. Int. Ed. 2012, 51, 6094.

(22) We have already employed triflate $\mathbf{1 7}$ as an efficient precursor of tetraphenylanthracyne $\mathbf{2 0}$, in the synthesis of large benzofused acenes: Rodríguez-Lojo, D. R.; Pérez, D.; Peña, D.; Guitián, E. Chem. Commun. 2015, 51, 5418.

(23) Pentacene and larger acenes are paradigmatic organic semiconductors, although their por solubility and low stability limit their application: Anthony, J. E. Angew. Chem. Int. Ed. 2008, 47, 452.

(24) Xiao, J.; Divayana, Y.; Zhang, Q.; Doung, H. M.; Zhang, H.; Boey, F.; Sun, X. W.; Wudl, F. J. Mat. Chem. 2010, 20, 8617 . 
Straightforward synthesis of novel acene-based aryne precursor

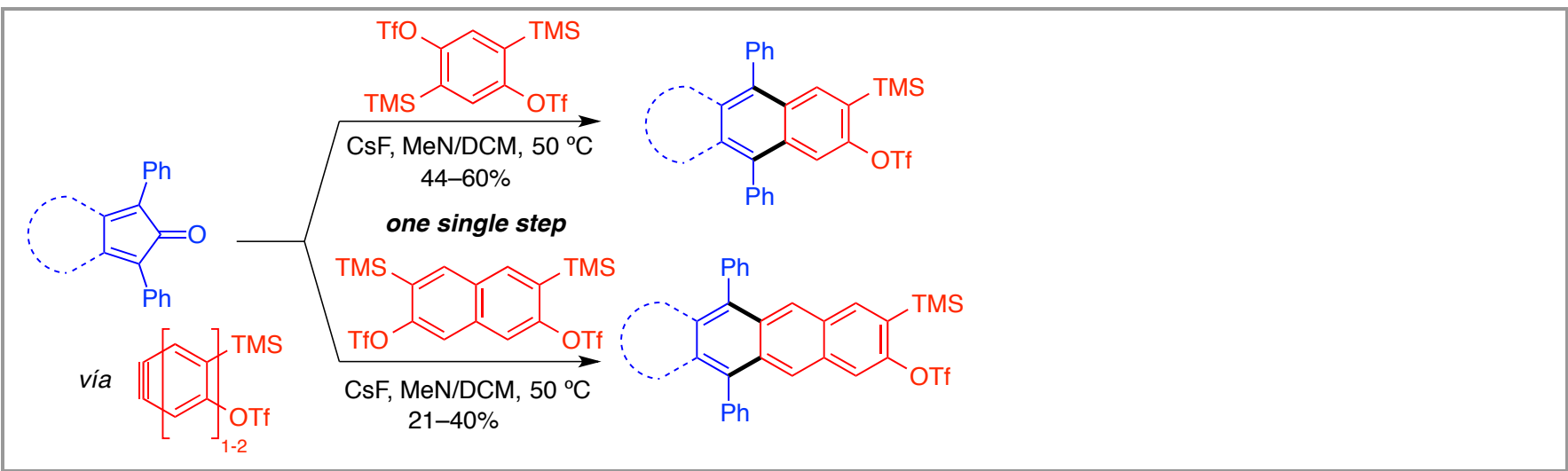

\section{Manuscript submission checklist}

- Statement of significance of work.

- Full mailing address, telephone, and fax numbers and e-mail address of the corresponding author.

- Graphical abstract.

- 5 key words.

- Original Word file.

- Original graphics files.

Proceed to submit your article via our online submission system at http://mc.manuscriptcentral.com/synlett. 